\title{
AUSSDA - DAS SOZIALWISSENSCHAFTLICHE DATENARCHIV MIT EUROPÄISCHER KOMPONENTE
}

\section{von Veronika Heider}

Zusammenfassung: Mit AUSSDA - The Austrian Social Science Data Archive wird eine Infrastruktureinrichtung für Sozialwissenschaften in Österreich aufgebaut. In diesem Beitrag werden zum einen organisatorische Eckdaten vorgestellt wie der Aufbau und die Zielgruppe der Organisation sowie (inter)nationale Kooperationen, zum anderen werden aber auch herausfordernde Aspekte der Archivarbeit präsentiert, wie die Weiterentwicklung des digitalen Archivs und Datenakquise und-nachnutzung.

Schlüsselwörter: Repositorien; Forschungsdaten; Sozialwissenschaften; digitales Archiv; Datennutzung; Datenzitation; DOI; Anonymisierung; Metadaten; Nutzungslizenzen; Datenmanagementplan

\section{AUSSDA - THE AUSTRIAN SOCIAL SCIENCE DATA ARCHIVE WITH A EUROPEAN COMPONENT}

Abstract: AUSSDA - The Austrian Social Science Data Archive is being established as an infrastructure for the social sciences in Austria. In this article basic organizational information like the structure and the target groups of the organization as well as (inter)national partnerships will be presented. In addition, some of the challenges facing the new archive will be outlined, concerning the development of the digital archive as well as data acquisition and data reuse.

Keywords: repositories; research data; social sciences; digital archive; data usage; data citation; DOI; anonymization; metadata; licenses; data management plan

DOI: http://doi.org/10.31263/voebm.v71i1.1996

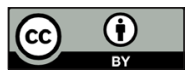

Dieses Werk ist lizenziert unter einer

Creative-Commons-Lizenz Namensnennung 4.0 International 


\section{Inhalt}
1. Einleitung
2. Aufbau und Governance
3. Archivierung sozialwissenschaftlicher Daten
4. Die technische Lösung
5. Zukünftige Entwicklungen

\section{Einleitung}

Ende 2016 nahm AUSSDA - The Austrian Social Science Data Archive seine Arbeit auf und begann mit dem Aufbau einer Forschungsinfrastruktur für die Sozialwissenschaften in Österreich. „Wir machen sozialwissenschaftliche Daten zugänglich und nachnutzbar, für Wissenschaft und Gesellschaft" (AUSSDA 2018). Anhand dieses Mission Statements wird bereits klar, dass AUSSDA nicht nur Forschenden zur Seite stehen will, sondern auch Hilfestellung leistet, nachdem Daten archiviert wurden, um die Nachnutzung der Daten durch eine breitere Öffentlichkeit zu fördern.

\section{Aufbau und Governance}

Nachdem das Wiener Institut für sozialwissenschaftliche Dokumentation und Methodik (WISDOM) 2013 seine Pforten schloss, gab es in Österreich kein Datenarchiv für die Sozialwissenschaften mehr. Einem engagierten Team von Professorinnen und Professoren ist es zu verdanken, dass das damalige Bundesministerium für Wissenschaft, Forschung und Wirtschaft (heute Bundesministerium für Bildung, Wissenschaft und Forschung) die Core Facility AUSSDA ins Leben gerufen hat. Neben dem Hauptstandort an der Universitätsbibliothek Wien sind weitere Standorte an der Karl-Franzens-Universität Graz und der Johannes Kepler Universität Linz zu finden. Da diese drei Institutionen gemeinsam Services zur Verfügung stellen, war im Aufbau eine gemeinsame Corporate Identity und Website wichtig.

In Newsbeiträgen berichtet AUSSDA auf der Website (https://aussda. at) laufend über nationale und internationale Kooperationen, wie jene mit dem Consortium of European Social Science Data Archives (CESSDA $E I^{1}{ }^{1}$. Das ständig anwachsende CESSDA ERIC vernetzt die Mitglieder miteinander und erarbeitet mit ihnen europaweit nutzbare Services. Auf 
nationaler Ebene gibt es zwei wichtige Gremien der Zusammenarbeit. Einerseits treffen sich Repräsentantinnen und Repräsentanten der Partnerinstitutionen (bestehend aus den Universitäten Wien, Linz, Graz und dem Bundesministerium für Bildung, Wissenschaft und Forschung) laufend um Richtungsentscheidungen zu treffen. Andererseits steht ein nationaler Beirat dem AUSSDA-Team zur Seite, in dem Vertreterlnnen von sieben Universitäten sowie Fördergebenden (FFG und FWF) und anderen Forschungseinrichtungen (wie IHS, ÖAW, Statistik Austria und WIFO) als BotschafterInnen für AUSSDA tätig werden. Ein internationaler Beirat, der durch Repräsentantlnnen europäischer Datenarchive besetzt wird, unterstützt bei technischen Belangen des Archivierens um AUSSDA stets auf dem aktuellsten Stand eines digitalen Repositoriums zu halten.

Weitere innerösterreichische Kooperationen bestehen zu dem Austrian Centre for Digital Humanities (ACDH) an der Österreichischen Akademie der Wissenschaften (ÖAW), dem institutionellen Archiv der Universität Wien (Phaidra), der Statistik Austria und der Universitätsbibliothek Innsbruck. Neben Forschungseinrichtungen sind auch disziplinspezifische Zeitschriften wie die SWS Rundschau, die Zeitschrift der Sozialwissenschaftlichen Studiengesellschaft, wichtige KooperationspartnerInnen für AUSSDA.

Ein großer Meilenstein, bei dem die Kooperationspartnerlnnen, Forschende und Medienvertreterlnnen zusammenkamen, war die öffentliche Vorstellung von AUSSDA und der Launch des digitalen Archivs „AUSSDA Dataverse“ im November $2017^{2}$. Für die Veranstaltung konnten spannende RednerInnen gewonnen werden: Neben einem Vortrag über „Data-linking"von Cees van der Eijk (University of Nottingham) und einem weiteren Vortrag „Open Science, Dark Knowledge“ von Klement Tockner (Präsident FWF) sowie der Vorstellung von CESSDA ERIC durch Ron Dekker (Direktor CESSDA ERIC), stand vor allem die Präsentation der Services und des Archivs von AUSSDA im Mittelpunkt.

\section{Archivierung sozialwissenschaftlicher Daten}

Die Services von AUSSDA richten sich an Sozialwissenschaftlerlnnen verschiedener Disziplinen. Nicht nur Forschende der Politikwissenschaft, Soziologie, Kommunikationswissenschaft oder den Bildungswissenschaften können AUSSDA nutzen, sondern auch Vertreterlnnen anderer sozialwissenschaftlicher und interdisziplinärer Studien können ihre Daten bei AUSSDA sicher speichern. In der Anfangsphase werden vor allem quanti- 


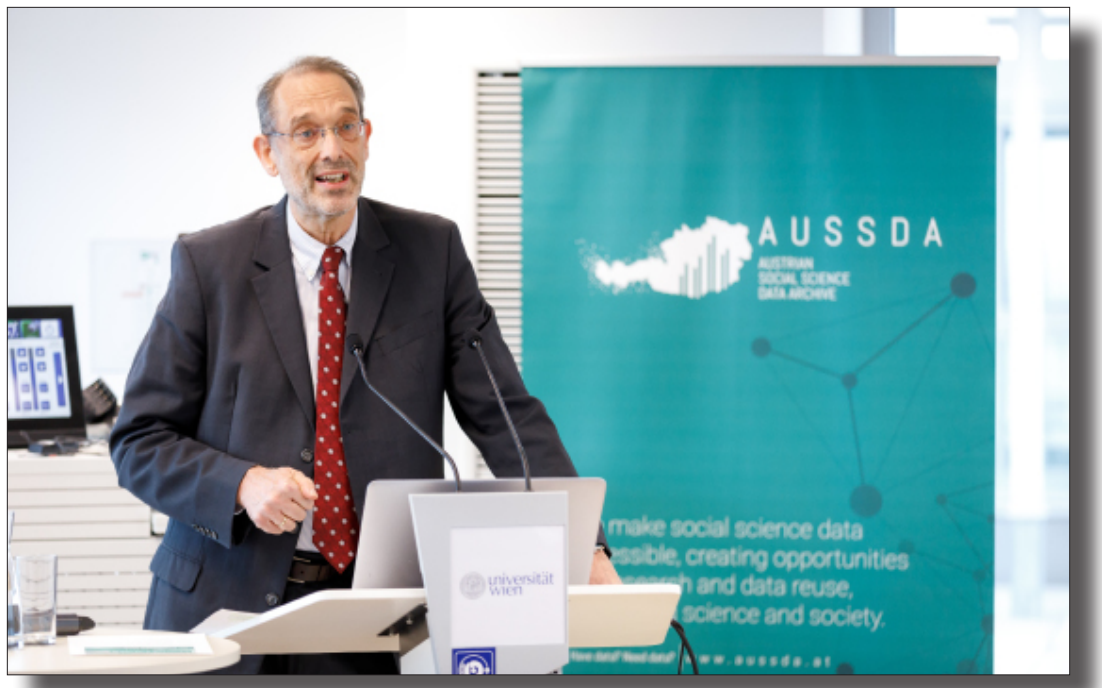

Abb. 1: Grußworte von Heinz Faßmann bei der ersten öffentlichen AUSSDA Präsentation (๔) AUSSDA/derknopfdruecker.com)

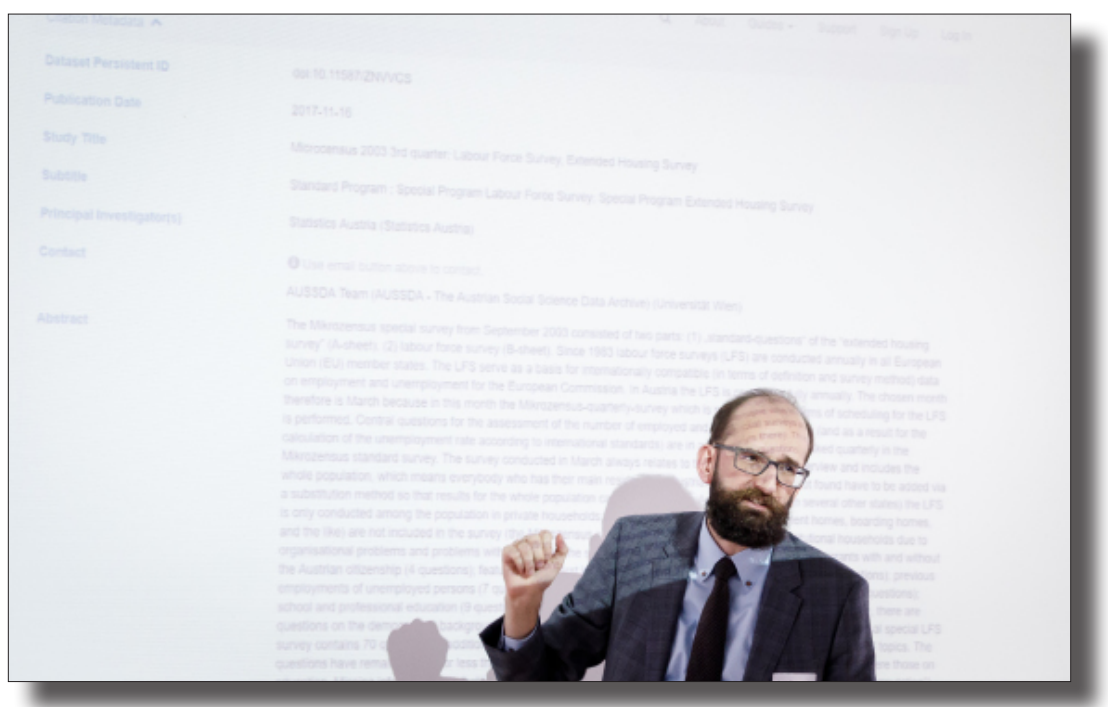

Abb. 2: Lars Kaczmirek präsentiert das AUSSDA Dataverse (๔ AUSSDA/derknopfdruecker. com) 
tative Daten archiviert, in einer späteren Phase wird auch die Aufnahme qualitativer Daten in den AUSSDA-Bestand angedacht.

Wissenschafterlnnen können AUSSDA in jedem Schritt des Forschungszyklus kontaktieren. Schon bei der Einreichung eines Forschungsantrags können Beratungen zu Datenmanagementplänen gegeben und damit der Grundstein für eine spätere erfolgreiche Datennachnutzung gelegt werden. Forschende können sich über Archivierungsmöglichkeiten informieren, da AUSSDA unterschiedliche Lizenzen für unterschiedliche Nachnutzungsszenarien anbietet. Wollen Forschende ihre Daten bei AUSSDA archivieren, müssen sie sich entscheiden, wie offen diese zur Verfügung gestellt werden sollen. Die Empfehlung von AUSSDA lautet, nach dem Leitsatz der Europäischen Kommission, „so offen wie möglich aber so geschlossen wie nötig“ (European Commission 2016). Im optimalen Fall wird ein Open-Access-Datensatz über das digitale Archiv zur Verfügung gestellt und mithilfe einer Lizenz wie der Creative Commons Namensnennung 4.0 (CC BY) frei verfügbar. Soll der Datensatz Open Access zur Verfügung stehen, sind Anonymisierungsfragen besonders wichtig. Mit der neuen Datenschutz-Grundverordnung (DSGVO) müssen auch Archive ihre Bedingungen anpassen und Sammlungen auf personenbezogene Daten hin überprüfen, wenn die Nachnutzung nicht auf wissenschaftliche Zwecke beschränkt werden soll.

Nachdem die Entscheidung für eine bestimmte Lizenz gefallen ist, erhält AUSSDA die Daten und Dokumentationen. Sie werden geprüft und kontrolliert, dabei werden die Nachvollziehbarkeit und der Stand der Anonymisierung überprüft und gegebenenfalls nachgebessert. Nicht nur finale Datensätze aus Projekten können zur Verfügung gestellt werden, sondern auch Replikationsdaten für Artikel, die in Fachzeitschriften veröffentlicht wurden, können bei AUSSDA archiviert werden. Jeder Eintrag im AUSSDA-Archiv bekommt als Persistent Identifier einen Digital Object Identifier (DOI). Damit erhöht sich die Auffindbarkeit von Daten und Dokumentation und die betreffenden Forschungsdaten können bei einer Nutzung in einer Publikation einheitlich zitiert werden.

Zu den ArchivierungspartnerInnen zählen zahlreiche große Forschungsprojekte in Österreich, unter anderem die Austrian National Election Study (AUTNES), der Soziale Survey Österreich (SSÖ), das International Social Survey Programme (ISSP) und die Plattform für Umfragen, Methoden und empirische Analysen (PUMA). Die Daten sind zum Teil bereits im Archiv verfügbar oder im Bearbeitungsprozess. Zudem begleitet AUSSDA auch Projekte, die sich mit neuen Datenarten beschäftigen, wie ein Projekt zu „big data“ in den Sozialwissenschaften. 


\section{Die technische Lösung}

Wie bereits oben erwähnt ist das digitale Archiv, das AUSSDA Dataverse, bereits unter https://data.aussda.at erreichbar. AUSSDA setzt auf die Open Source-Anwendung Dataverse (https://dataverse.org/), die von der Harvard Universität entwickelt wurde. Es wird zwischen Datensätzen und sogenannten Dataverses unterschieden: der Inhalt eines Datensatzes besteht aus Datendateien, Dokumentationen und Metadaten, während ein Dataverse eine Reihe von Datensätzen beheimaten kann.

Es besteht auch die Möglichkeit, Ergebnisse eines Projekts, einer Forschendengruppe oder einer Fachzeitschrift zusammenzufassen und gemeinsam in einem Dataverse sichtbar zu machen. Will eine Fachzeitschrift also Replikationsdaten von Publikationen im AUSSDA Dataverse zur Verfügung stellen, bekommt es ein eigenes Dataverse im Archiv. Im Dataverse der Zeitschrift, welches mittels Plugin auch auf der Website der Fachzeitschrift eingebettet werden kann, bekommt dann jeder Replikationsdatensatz einer Publikation einen eigenen Datensatz-Eintrag, der die beschreibenden Metadaten, die Datendateien und die Dokumentationen beinhaltet.

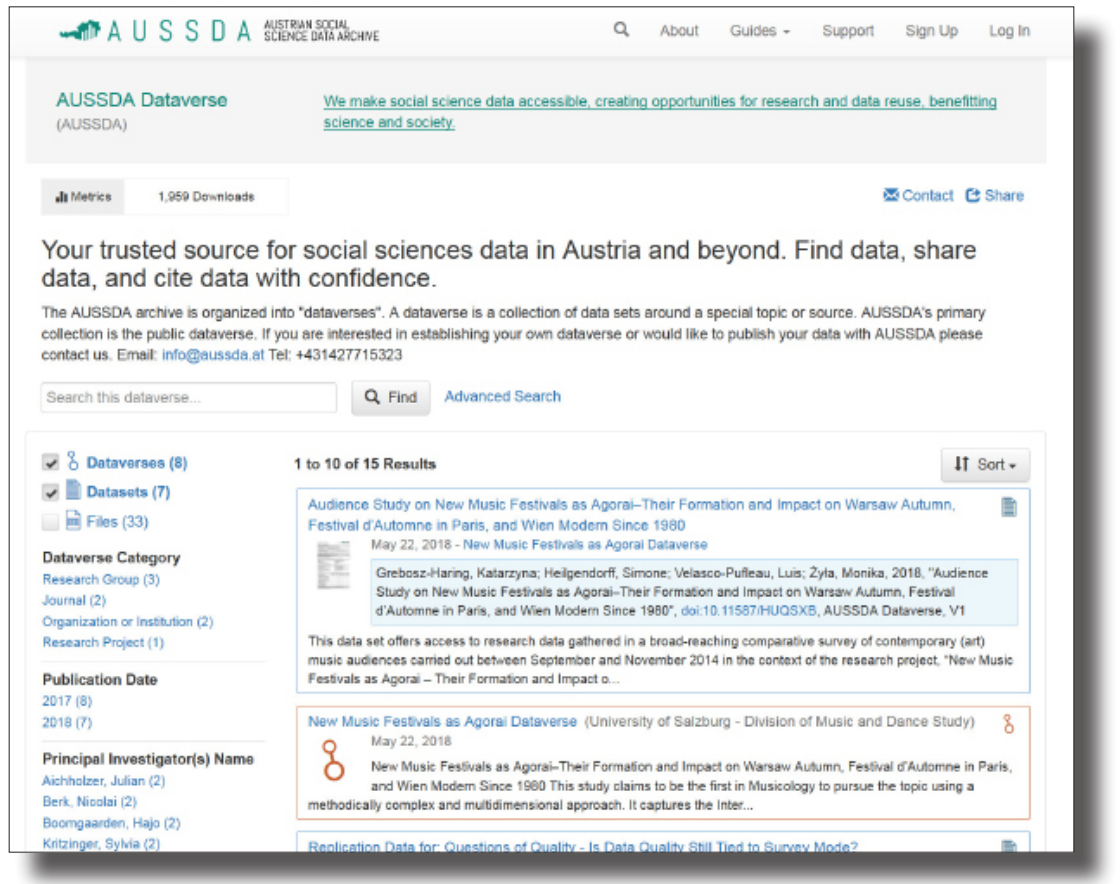

Abb. 3: Das digitale Archiv AUSSDA Dataverse, erreichbar unter https://data.aussda.at 
Die Weiterentwicklung der Anwendung ist ein wichtiger Teil der Arbeit von AUSSDA. In dem Projekt „DataverseEU“ werden gemeinsam mit den europäischen Partnerlnnen von CESSDA ERIC diverse Erweiterungen entwickelt, wie etwa eine mehrsprachige Benutzendenoberfläche. CESSDA ERIC ist auch für die von AUSSDA verwendeten Standards relevant. Im Rahmen eines CESSDA-Projekts werden gemeinsame Metadatenstandards festgelegt, um über nationale Grenzen hinweg Daten in einem europäischen Datenkatalog auffindbar und nachnutzbar zu machen. Des Weiteren werden mit der CESSDA Topic Classification und dem European Language Social Science Thesaurus (ELSST) einheitliche Themen und Schlagwörter vergeben.

\section{Zukünftige Entwicklungen}

Während schon viele Herausforderungen wie der Aufbau der Governance-Struktur, der technologischen Strukturen (Website und Archiv) und die Entwicklung wichtiger Arbeitsprozesse in der Datenaufbereitung erfolgreich bestanden wurden, gibt es noch zahlreiche Aufgaben für das AUSSDA-Team. Eines der Kernziele für das Jahr 2018 ist die Datenakquise. Neben der Informationsvermittlung durch den nationalen Beirat, den Besuch fachrelevanter Konferenzen und Veröffentlichungen in Fachzeitschriften verschiedener Disziplinen, besucht das AUSSDA-Team auch aktiv Standorte, um Interessierten das Archiv vorzustellen. Im Moment sind Veranstaltungen an Universitäten, Fachhochschulen, Pädagogischen Hochschulen und weiteren Forschungseinrichtungen geplant.

Weitere Aktivitäten, die an die breitere Öffentlichkeit gerichtet sind, werden im Moment mitgedacht und nach 2018 eine wichtigere Rolle einnehmen. Denn nicht nur Forschende und WissenschafterInnen können AUSSDA kontaktieren und die Services nutzen. Während für diese Nutzerinnen und Nutzer die Datensätze in den üblichen Formaten der sozialwissenschaftlichen Forschung (Stata, SPSS, RData) zur Verfügung gestellt werden, wird durch die Verfügbarkeit der Daten in nicht-proprietären Formaten (wie etwa im tab delimited Format) eine breitere Nutzendengruppe erreicht. Datensätze, die mit einer offenen Lizenz archiviert wurden, können von interessierten Personen einfach und unkompliziert heruntergeladen und verwendet werden. Die Öffentlichkeitsarbeit umfasst neben Schulungen, Roadshows und Datenweitergabe auch Social Media Aktivitäten. Da viele für AUSSDA relevante Akteurlnnen in Österreich auch der Twitter-Community angehören, ist diese Plattform neben der AUSSDA-Website ein 
wichtiges Medium, das inhaltlich bespielt wird. Auf der Website und über Twitter werden auch Veranstaltungen, neue Datensätze und Services vorgestellt und beworben.

\section{国 Neuigkeiten}

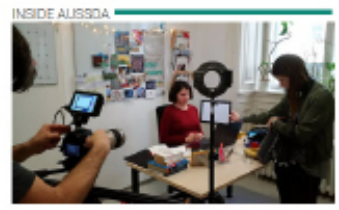

AUSSDA on video

Wir können stolz das Ergebnis einer Kooperation mit dern

Wissenstransferzentrum Ost der Uni Wien und dem TV-Sender Okto präsentieren: ein kurzes Video über die Arbeit von AUSSDA. Wir reichten unsere Idee ein, schrieben ein kurzes Drehbuch, identifizierten Drehorte, spielten selbst in den Hauptrollen und am Donnerstag fand die Präsentation unseres Werks im Top... Mehr
SEAVCE

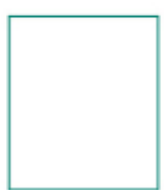

Image not available due to copyright restrictions

\section{Die Geschichte von Creative} Commons

Urheberrechtsgesetze beschränken, wie Daten und andere Arbeiten geteilt und wiederverwendet werden konnen. Creative Cammons (CC) Lizenzen Iosen diese Probleme und machen das volle Potenzial des Internets ausschöpfbar.

Mehr
$1234 \ldots H$

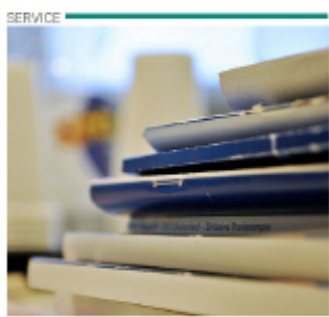

AUSSDA gibt Replikationsdaten ein Zuhause

Transparenz und Replizierbarkeit sind wichtige Schlagworte, wenn es um das Zustandekommen von

Forschungsergebnissen geht. Um Resultate eines Artikels einer Fachzeitschrift nachvollziehbar zu machen, bietet AUSSOA ein Replikationsdatenservice an. Mit einigen Journals bestehen bereits

Abb. 4: Newsbeiträge auf der AUSSDA-Website (https://aussda.at/)

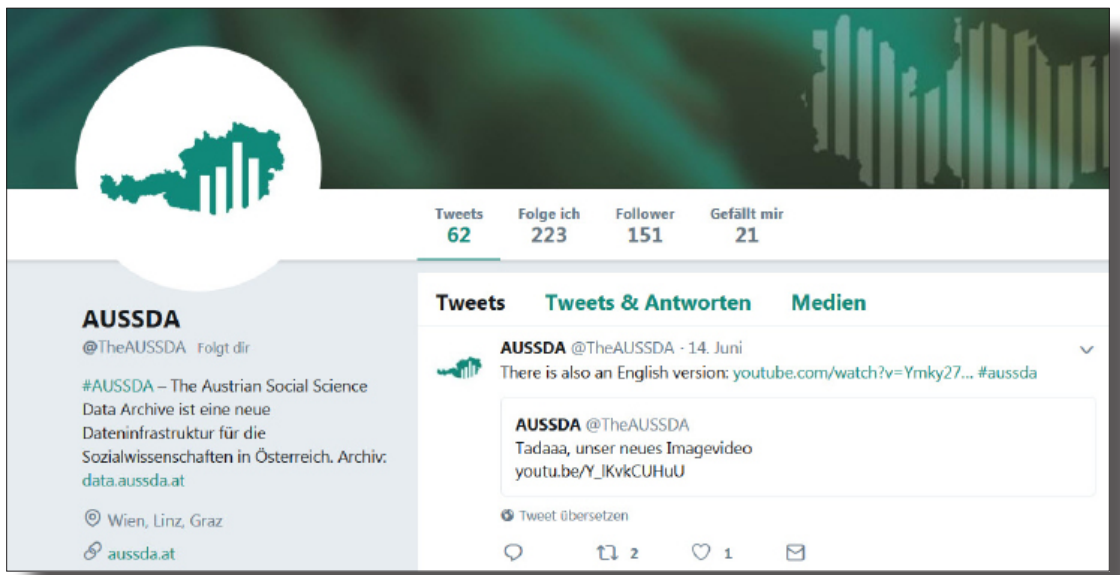

Abb. 5: Der Twitter-Account @TheAUSSDA 
Um die Inhalte, die bei AUSSDA archiviert werden, für ein größeres Publikum zugänglich zu machen, werden in der Zukunft auch Visualisierungen eine wichtige Rolle spielen. So können Inhalte schneller greifbar und breiteren Nutzendengruppen zugänglich gemacht werden. Zukünftige Entwicklungen sollen auch immer die Benutzenden miteinbeziehen. Als Forschungsinfrastruktur hat AUSSDA klar einen Fokus auf die User Experience, denn nur in einem produktiven Miteinander zwischen Wissenschaft, Gesellschaft und Archiv kann ein nachhaltiges Bestehen von AUSSDA garantiert werden.

\author{
Veronika Heider, BA BA MA \\ ORCID: http://orcid.org/0000-0001-8480-0029 \\ Universität Wien, AUSSDA - The Austrian Social Science Data Archive \\ E-Mail:veronika.heider@univie.ac.at
}

\title{
Literatur
}

AUSSDA (2018) AUSSDA - the Austrian Social Science Data Archive, verfügbar unter: http://www.aussda.at (7. 3. 2018).

European Commission (2016) H2020 Programme. Guidelines on FAIR Data Management in Horizon 2020, verfügbar unter: http://ec.europa.eu/ research/participants/data/ref/h2020/grants_manual/hi/oa_pilot/ h2020-hi-oa-data-mgt_en.pdf

1 Erfahren Sie mehr über CESSDA ERIC auf der Website https://www. cessda.eu/.

2 Das Programm sowie den Mitschnitt des Events findet man auf der AUSSDA Website unter diesem Link: http://aussda.at/ueber-aussda/ events/moving-social-sciences-ahead/. 